\title{
Low Beat-Noise Polarized Tunable Fiber Ring Laser
}

\author{
H. L. Liu, H. Y. Tam, Senior Member, IEEE, W. H. Chung, P. K. A. Wai, Senior Member, IEEE, and N. Sugimoto
}

\begin{abstract}
A low beat-noise highly polarized tunable fiber ring laser is proposed and experimentally demonstrated by using a Fabry-Pérot laser diode (FP-LD), which acts as a high-pass filter to suppress both the beat noise and TM-polarized light. The wavelength of the fiber ring lasers can be tuned from 1536.82 to $1570.72 \mathrm{~nm}$ in 1.12-nm steps and its maximum output power is about $+3 \mathrm{dBm}$. The degree of polarization and extinction ratio of the laser output are about $99 \%$ and $60 \mathrm{~dB}$, respectively. The beat-noise with and without the FP-LD was reduced dramatically by $50 \mathrm{~dB}$.
\end{abstract}

Index Terms-Laser noise, polarization, ring lasers.

\section{INTRODUCTION}

$\mathbf{F}$ IBER ring lasers have attracted tremendous interest because of their many important applications in fiber-optic test and measurement [1], fiber communications [2], and fiber sensor systems [3]. This is because fiber ring lasers exhibit wide tunable range, narrow linewidth, and could be tuned at high speed allowing fast component characterization. However, intensity noise from these fiber ring structures, especially beat-noise which is generated as a result of beating effect between the lasing longitudinal modes, could severely limit the lasers' applications. Zhang et al. proposed a compound-ring cavity to reduce the beat-noise [4]. Due to the short ring-length, the dual-coupler fiber ring acts as a small free spectral range (FSR) etalon filter and combines with the tunable optical bandpass filter to select one longitudinal mode. However, it needs PZT to accurately control the length of the cavity. Recently, the reduction of beating noise using unpumped erbium-doped fiber (EDF) as saturable absorber, operating as narrow-band filter was reported by several groups [5]-[7]. However, this method is sensitive to the environment due to the relatively long fiber length.

In this letter, we propose a novel method to suppress the beatnoise fiber ring laser using a Fabry-Pérot laser diode (FP-LD). The beat-noise of fiber ring lasers is primarily in the low-frequency region of about $10 \mathrm{MHz}$ due to the long ring cavity

Manuscript received October 18, 2005; revised December 21, 2005. The work described in this letter was supported in part by grants from the Research Grants Council of the Hong Kong Special Administrative Region, China (Project G-YE16) and in part by Asahi Glass Corp., Japan. The work of P. K. A. Wai was supported by the Research Grant Council of the Hong Kong Special Administrative Region, China (Project A-PF79).

H. L. Liu, H. Y. Tam, and W. H. Chung are with the Photonics Research Centre, Department of Electrical Engineering, The Hong Kong Polytechnic University, Hung Hom, Kowloon, Hong Kong, China (e-mail: eehlliu@polyu.edu.hk).

P. K. A. Wai is with the Photonics Research Centre, Department of Electronics and Information Engineering, The Hong Kong Polytechnic University, Hung Hom, Kowloon, Hong Kong, China.

N. Sugimoto is with the Asahi Glass Co., Ltd, Yokohama 221-8755, Japan.

Digital Object Identifier 10.1109/LPT.2006.871152

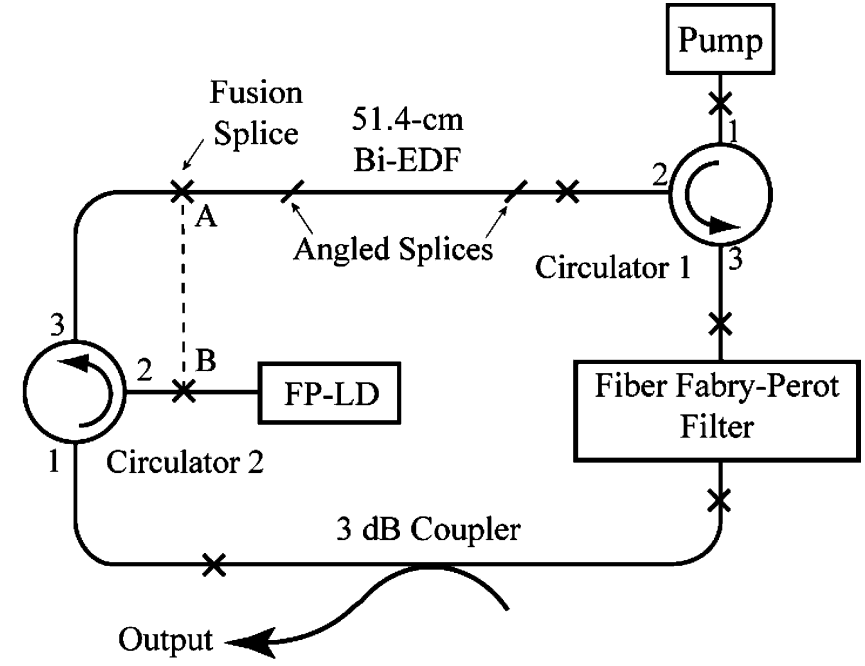

Fig. 1. Configurations of the highly polarized, low beat-noise, tunable fiber ring laser.

length which is typically of the order of several tens of meters. On the other hand, injected FP-LD has relatively fast carrier recovery rate $(<1$ nanosecond) and exhibits gain saturation effect; it can be employed as a high-pass filter to suppress the low-frequency noise [8]. Therefore, the introduction of an FP-LD into the fiber laser cavity can dramatically suppress the low-frequency intensity beat-noise of fiber ring lasers.

\section{EXPERIMENTAL SETUP}

Fig. 1 shows the configuration of the low beat-noise highly polarized traveling wave tunable fiber ring laser. The Bismuth oxide-based EDF (Bi-EDF) is pumped by one 1480-nm semiconductor laser diode via Port 1 of an optical circulator, which exhibits fairly flat passband in the wavelength range of 1460 to $1630 \mathrm{~nm}$. The length of the Bi-EDF is $51.4 \mathrm{~cm}$ long. The refractive index of the core and cladding of the Bi-EDF are 2.03 and 2.02, while the diameter of the core and cladding are 3.9 and $124.7 \mu \mathrm{m}$, respectively. The erbium concentration in the Bi-EDF is $6500 \mathrm{wt} \cdot \mathrm{ppm}$ and Boron and Lanthanum are codoped in the $\mathrm{Bi}_{2} \mathrm{O}_{3}$-based fiber to increase the pump efficiency. The peak absorption of the Bi-EDF at 1480 and $1530 \mathrm{~nm}$ are 141 and $219 \mathrm{~dB} / \mathrm{m}$, respectively. Both ends of the Bi-EDF was first angle spliced to high numerical aperture fiber (Nufern 980-HP fiber) before splicing to Port 2 (SMF-28 fiber) of Circulator 1 and to Port 3 (SMF-28 fiber) of Circulator 2, providing better mode field diameter matching. The splicing loss attained was less than $0.2 \mathrm{~dB}$ for the angled splices. The angled splices reduce the reflection in the laser cavity to less than $60 \mathrm{~dB}$.

A large FSR fiber Fabry-Pérot (FFP) filter was employed to tune the laser wavelength. The optical bandwidth and FSR of 


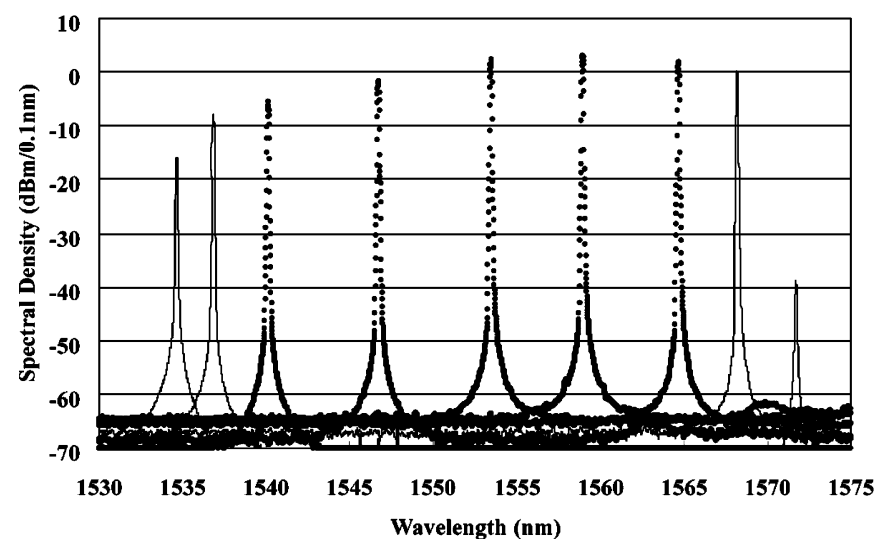

Fig. 2. Superimposed laser output spectra of the fiber ring laser measured by an OSA with $0.1-\mathrm{nm}$ resolution.

the FFP filter are about $50 \mathrm{pm}(\sim 6.25 \mathrm{GHz})$ and $110 \mathrm{~nm}$, respectively. Since the gain bandwidth of Bi-EDF is less than $110 \mathrm{~nm}$ [9], only one wavelength in the fiber ring laser cavity will be excited. The insertion loss of the FFP filter at the peak of the passband is about $2.2 \mathrm{~dB}$. The out-of-band reflection from both ends of the FFP filter is quite high. Circulator 1 (from Port 2 to Port 3) was also used as an optical isolator to reduce the reflection from the FFP filter back to the Bi-EDF as well as to ensure unidirectional operation of the ring laser. A double-channel planar-buried heterostructure FP-LD with cavity mode spacing and threshold current of $1.12 \mathrm{~nm}$ and $10.9 \mathrm{~mA}$, respectively, was inserted in the fiber cavity. The FP-LD was biased at $12 \mathrm{~mA}$, slightly above the threshold current, to realize the low beat-noise laser output. A 3-dB fused fiber taper was included in the laser cavity to provide the laser output.

\section{PeRformance AND Discussions}

Fig. 2 shows the superimposed output spectra of the fiber ring laser obtained from the output port of the 3-dB coupler. The spectra were measured by an optical spectrum analyzer (OSA) with 0.1-nm resolution. The fiber ring laser was pumped with $150 \mathrm{~mW}$ of optical power. If the filter wavelength is close to one of the FP-LD cavity modes, the FP-LD will be injection-locked, permitting gain to the input signal and lased at the wavelength determined by the filter peak transmission wavelength [10]. By varying the voltage applied to the FFP filter, the laser wavelength can be tuned from 1536.82 to $1570.50 \mathrm{~nm}$ in 1.12-nm steps. The tuning range and step are decided by the bandwidth and mode spacing of FP-LD, respectively. If FP-LD with broader bandwidth is available, wider tunable range could be achieved. Since the laser output was taken from the output of the FFP filter which filters the out-of-band amplified spontaneous emission, a large extinction ratio of about $60 \mathrm{~dB}$ was achieved, as shown in Fig. 2. The maximum output power of the fiber laser is about $+3 \mathrm{dBm}$. The measured linewidth of the fiber ring laser by a radio-frequency (RF) spectrum analyzer is shown in Fig. 3. It is measured by the delayed self-heterodyne method using an acoustooptics frequency shifter driven at $\sim 99.99-\mathrm{MHz}$ frequency, $25-\mathrm{km}$ delayed fiber, and $125-\mathrm{MHz}$ photodiode detector. Assuming that the laser spectrum is Lorentzian-shaped, the $3-\mathrm{dB}$ linewidth of the fiber ring laser is about $1.2 \mathrm{kHz}$, as shown in the inset of Fig. 3.

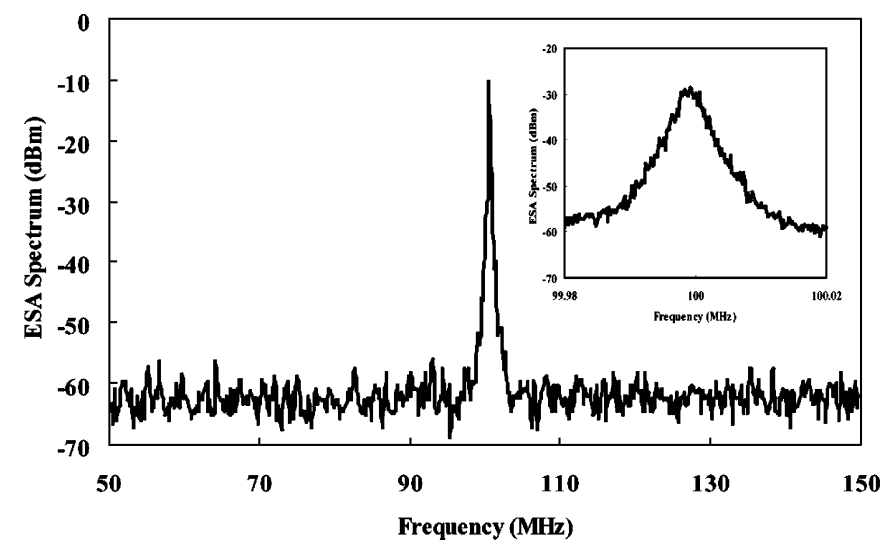

Fig. 3. Measured laser linewidth of the fiber ring laser with the delayed self-heterodyne method.

Since the double-channel planar-buried heterostructure of FP-LD favors the TE mode, the TE component of the injected signal would be amplified with its intensity clamped and stabilized by injection locking if the power of the TE component is above the injection-locking threshold of the FP-LD, while the TM component, however, is always suppressed. As a result, the laser output is highly polarized. To measure the degree of polarization (DOP) of the laser, the laser output was connected to a polarization controller, a polarizer and then to a photodetector. By varying the polarization states of the polarization controller, a maximum value of $23-\mathrm{dB}$ power differences was observed, which means that the DOP of the laser is about $99 \%$. The DOP can be calculated as

$$
\begin{aligned}
\mathrm{DOP} & =\frac{P_{\max }-P_{\min }}{P_{\max }+P_{\min }} \\
& =1-2 \frac{P_{\min }}{P_{\max }+P_{\min }} \approx 1-2 \frac{P_{\min }}{P_{\max }} .
\end{aligned}
$$

In (1), it is assumed that $P_{\min } \ll P_{\max }$. The RF spectra of the fiber ring laser with and without FP-LD, which is realized by connecting points $A$ and $B$ together (Fig. 1), were shown in Fig. 4(a) and (b), respectively. The RF spectra were measured with a $25-\mathrm{GHz}$ photodiode detector (NewFocus) that is connected to an RF electrical spectrum analyzer with $100-\mathrm{kHz}$ resolution. The input optical power to the photodetector is kept at about $-2 \mathrm{dBm}$. Without the FP-LD, the output of the laser exhibits a strong intensity noise, as shown in Fig. 4(a), which is generated by the beating effect of the multilongitudinal modes. The minimum beat frequency component is determined by the longitudinal mode spacing, which depends on the ring cavity length. In this case, $\sim 18-\mathrm{MHz}$ beat frequency component was observed which indicate that the ring cavity length is about $11 \mathrm{~m}$. As shown in Fig. 4(b), with the FP-LD included in the cavity, the intensity of the beat noise of the laser was suppressed dramatically from about $-30 \mathrm{dBm}$ to less than $-80 \mathrm{dBm}$.

\section{CONCLUSION}

We have proposed and experimentally demonstrated a low beat-noise highly polarized tunable fiber ring laser, which is realized by employing an FP-LD which acts as a high-pass filter to suppress the low-frequency beat-noise. The laser configuration permits wavelength tuning over $34 \mathrm{~nm}$, from 1536.82 to 


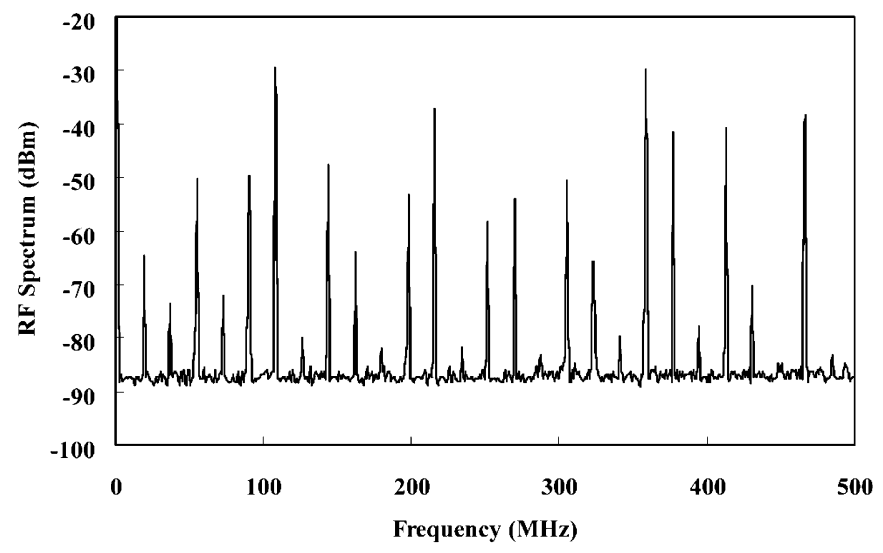

(a)

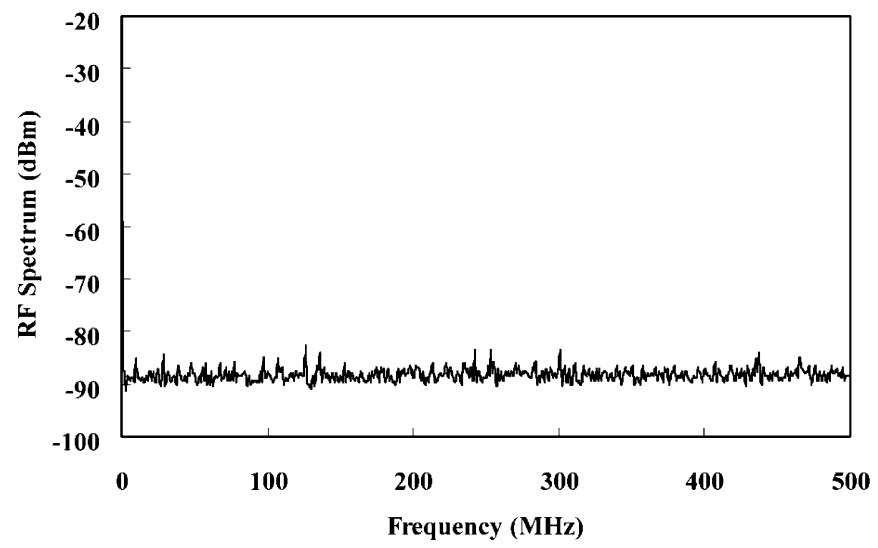

(b)

Fig. 4. RF spectra of the laser output (a) without and (b) with FP-LD.

$1570.50 \mathrm{~nm}$ in 1.12-nm steps. The maximum output power of the laser is about $+3 \mathrm{dBm}$ and exhibit a very high extinction ratio of about $60 \mathrm{~dB}$. The beat-noise was reduced significantly by about $50 \mathrm{~dB}$.

\section{REFERENCES}

[1] A. Bellemare, M. Karásek, C. Riviere, F. Babin, G. He, V. Roy, and G. W. Schinn, "A broadly tunable erbium-doped fiber ring laser: Experimentation and modeling," IEEE J. Sel. Topics Quantum Electron., vol. 7 , no. 1, pp. 22-29, Jan. 2001.

[2] T. Haber, K. Hsu, C. Miller, and Y. Bao, "Tunable erbium-doped fiber ring laser precisely locked to the $50-\mathrm{GHz}$ ITU frequency grid," IEEE Photon. Technol. Lett., vol. 12, no. 11, pp. 1456-1458, Nov. 2000.

[3] Y. Yu, H. Y. Tam, L. F. Lui, and W. H. Chung, "Fiber-laser-based wavelength division multiplexed fiber Bragg grating sensor system," IEEE Photon. Technol. Lett., vol. 13, no. 7, pp. 702-704, Jul. 2001.

[4] J. L. Zhang, C. Y. Yue, G. W. Schinn, W. R. L. Clements, and J. W. L. Lit, "Stable single-mode compound-ring erbium-doped fiber laser," $J$. Lightw. Technol., vol. 14, no. 1, pp. 104-109, Jan. 1996.

[5] Y. Cheng, J. T. Kringlebotn, W. H. Loh, R. I. Laming, and D. N. Payne, "Stable single-frequency traveling-wave fiber loop laser with integral saturable-absorber-based tracking narrow-band filter," Opt. Lett., vol. 20, no. 8, pp. 875-877, Apr. 1995.

[6] Y. W. Song, S. A. Havstad, D. Starodubov, Y. Xie, A. E. Willner, and J. Feinberg, "40-nm-wide tunable fiber ring laser with single-mode operation using a highly stretchable FBG," IEEE Photon. Technol. Lett., vol. 13, no. 11, pp. 1167-1169, Nov. 2001.

[7] H. X. Chen, F. Babin, M. Leblanc, and G. W. Schinn, "Widely tunable single frequency erbium-doped fiber lasers," IEEE Photon. Technol. Lett., vol. 15, no. 2, pp. 185-187, Feb. 2003.

[8] L. Xu, I. Glesk, D. Rand, V. Baby, and P. R. Prucnal, "Suppression of beating noise of narrow-linewidth erbium-doped fiber ring lasers by use of a semiconductor optical amplifier," Opt. Lett., vol. 28, no. 10, pp. 780-782, May 2003.

[9] H. L. Liu, H. Y. Tam, W. H. Chung, P. K. A. Wai, and N. Sugimito, "La-codoped bismuth-based erbium-doped fiber ring laser with 106-nm tuning range," IEEE Photon. Technol. Lett., vol. 17, no. 2, pp. 297-299, Feb. 2005

[10] L. Y. Chan, W. H. Chung, P. K. A. Wai, B. Moses, H. Y. Tam, and M. S. Demokan, "Simultaneous repolarization of two $10 \mathrm{~Gb} / \mathrm{s}$ polarizationscrambled wavelength channels using a mutual-injection-locked laser diode," IEEE Photon. Technol. Lett., vol. 14, no. 12, pp. 1740-1742, Dec. 2002. 